\title{
A hybrid image denoising method based on clustering and PDE
}

\author{
Sonal Pandya ${ }^{1 *}$ and Ravindra Gupta ${ }^{2}$ \\ M.Tech Student, Computer Science and Engineering, SRK University, Bhopal, India ${ }^{1}$ \\ Professor, Computer Science and Engineering, SRK University, Bhopal, India ${ }^{2}$ \\ (C)2018 ACCENTS
}

\begin{abstract}
This paper provides an efficient method based on the combination of hierarchical clustering along with the capability of PDE, FFT and color domination. Then for the edge point selection decomposition has been performed. It is applied with the clustering mechanism so that data points are separated. Then by similarity ranking alike data points are separated and decomposed. By this process, noise can be separated and other image proprieties along with the alikeness are separated. The color domination, PDE and FFT combination have been applied. This is applied on the data obtained from the previous process. This step provides the color based separation and error filtration. PSNR values have been used for the comparative study. The obtained results have higher PSNR then the previous approaches shows the effectiveness of our approach.
\end{abstract}

Keywords

Hybrid method, PDE, FFT, PSNR.

\section{Introduction}

Image denoising have been explored by different researchers now a days and it is significantly increase the era and application scenario in the today's world [1-3]. It has been used widely in the area for fulfilling the noise removal application in case of several aspects. In the current Gaussian noise and impulse noise $[4,5]$. The pixel position and other effects have been induced through the Gaussian noise. The ratio of different approaches and the directions have been discussed and allowed in the direction of filtering and the enhancement of the approaches for the methods in different area considered for the application area [6]. It also includes the nature enhancement and then it is correlated according to the pixels. Ordinarily, the tumult identifies with a higher repeat territory, the primary picture is essentially arranged in the lower repeat zone, and a part of the image focal points are arranged in the high repeat band [7-10]. The repeat change based denoising methodology typically uses the Fourier change to change over the image from the spatial space to the repeat territory, and a while later removes the tumult section by applying a channel with a specific cutoff repeat, in conclusion uses the switch Fourier change to get a smooth picture[11].

*Author for correspondence

484
In any case, these exercises are repetitive and they moreover darken the focal points of the image. Writing proposed that the Fourier change gives recurrence data yet the wavelet change gives the confinement in both time area. Multi-goals and the sparsity is the upside of wavelet change and in addition the vitality compaction [12-14].

There are a couple of strategies which are on a to a great degree basic dimension stress with denoise photo data, for instance, averaging channel, middle channel, Gaussian channel and partial differential equation (PDE) approach. If we examine the properties of good pictures then it will be with the less racket and reason for containment the dull or cloud decreasing is the major factor. The PDE approach is much sensible and applies in a few investigation like $[15,16]$. In any case, it is basically increasingly profound in case we apply fourth demand fragmentary differential condition. Employments of the PDE models can be generally found in a wide level of picture recovery errands, for instance, denoising and change [17, 18] shading picture managing and confirmation. This gives us the future learning or works with the forward demand inadequate differential condition with a close demand toward cloud diminish. 


\section{Related work}

In 2011, In 2017, Chithra and Santhanam [19] proposed a hybridization algorithm based on filteration. They have used Gaussian noise in computer tomography (CT). It is useful in enhancing the nature of the picture. Their technique is the hybridization of modified median wiener filter (MMWF) and absolute difference and mean (ADMF) filter. It is compared and evaluated based on the triangular and direction-based filter (TDBF), discrete wavelet transform using total variation (DWTTV), edge preserving hybrid filter (EPHF) and ADMF filter. The outcome demonstrates the proposed strategy is better in terms of peak signal to noise ratio (PSNR) and mean square error (MSE).

In 2017, Soni and Kirar [20] talks about the troubles found in commotion departure. Over the range of ongoing decades, various types of complain diminishing systems have been conveyed. They surveys the change based denoising procedures and plays out their equivalent examination. They put results of various techniques including general ridgelets and curvelets, Experimental Mode Decomposition and Empirical ridgelets and curvelets. Quantitative extents of examinations are appeared at PSNR.

In 2017, Pang [21] recommended that the wavelet rotting levels and the decision of the thresholding limit may impact the execution of picture denoising. They have shown another methodology for the wavelet decaying levels ID using the 2D Haar wavelet thresholding strategy. It utilizes the standard deviation estimations of the sub-social events to see whether the flag noteworthiness is solid or slight in the high recurrent sub-packs after the 2D Haar wavelet change. Being developed, another thresholding limit is proposed which accomplishes better denoising execution to the degree PSNR and MSE than the delicate thresholding framework. Particularly, at high tumult levels, the proposed new thresholding system overcomes hard thresholding, touchy thresholding and semi-delicate thresholding techniques.

In 2017, Yang and Liu [22] suggested that the fluorescence molecular imaging tomography (FMT) has the upsides of early intervention, straightforwardness and basic use. FMT can gain the task space of fluorophore in body without cutting the body or setting something into the body. In any case, fluorescence sub-atomic picture is to an extraordinary degree delicate to different sort of unsettling influences, for example, autofluorescence, establishment racket a few different turmoil ridden situations. The fluorescence hail is the real area in fluorescence picture, the power of auto fluorescence and other foundation signals are all things considered low in the examination result. This technique dispose of high recurrent objects by Gaussian smoothing in rehash an area, by at that point, to expel the significant sections of the fluorescence developments by wavelet change, at last, k-suggests gathering is used to detach the basic part and foundation of the fluorescence picture. Primer works out as expected demonstrate that the proposed strategy is reasonableness, it could get the fluorescence hail while wipe out the foundation racket, and enlargement the possibility of fluorescence picture.

In 2017, Ankarao et al. [23] proposed that the symmetrical coordinating calculation is financially savvy as far as computational unpredictability. This figuring gives a reaction for over chose in like manner, underdetermined frameworks by confining as far as possible utilizing littlest square. This work revolves around the enhancement of vocabulary which can be utilized to understand the sparsity based picture denoising issue. They built up the word reference least square arrangement exposed to thresholding conditions. Orthogonal matching pursuit (OMP) count avoids the affirmation of a similar atom in each complement, in light of the closeness of symmetrical property between the store and the molecule investigated the word reference. Along these lines, OMP figuring accomplishes right picture age. The proposed system is embraced on four standard test pictures, for example, Lena,Vessel, Barbara and Cameraman with various rackets, for example, salt and pepper hubbub, Gaussian whine and spot object with fluctuating the dimension of tumult level from $5 \%$ to $40 \%$. Gotten happens are assessed by the quality metric PSNR and separated and the present wavelet based melancholy picture denoising. The test assessment shows that the proposed framework is better material to remove the bit disarray and salt and pepper commotion when separated and the present wavelet based inadequate picture denoising.

In 2018, Vyas and Paik [24] provide the analysis and exploration of the methods based on the previous approaches.

In 2018, Liu et al. [25] recommended that the reproduction of the picture in the wake of denoising is a critical perspective. They have proposed a CNN demonstrate in profound learning for picture 
denoising. Differentiated and standard picture denoising methods, for instance, ordinary filtering, Wiener isolating and center filtering, the ideal position of using this $\mathrm{CNN}$ exhibit is that the parameters of this model can be streamlined through framework planning; however in customary picture denoising, the parameters of these are settled and can't be adjusted in the midst of the isolating, to be explicit, nonattendance of adaptivity.

In 2018, Mbarki et al. [26] proposed that the principle point of non-daze picture rebuilding is to evaluate the genuine picture accepting the haze. A noteworthy procedure in the filtering speculation used consistently for picture recovery is the Wiener channel. The detriment of this technique is the prerequisite for from the earlier learning of the corruption work, the darkened picture what's more, the quantifiable properties of the confusion method. In this work, a non-stun picture remaking figuring using the parametric wiener filtering and BM3D denoising framework has been proposed. Immediately, the debased picture is deconvoluted in Fourier space by parametric Wiener filtering, and from that point forward, it is smoothed by the BM3D method. Test outcomes are intriguing and engaging.

\section{Methods}

This paper provides the hybridization of hierarchical clustering, PDE, FFT and dominant color based selection with edge consideration. The process is started with the data pre-processing procedure for making it compliant for the programming. This process is performed according to the matrix arrangement, color based separation and data mosaicking. It also covers the measurement of color performance, sharpening and transformation. In this procedure the information development in the settled measurement for the further procedure has been defined. Last information pre-processed values have been gotten. The process is shown in Figure 1. Then denoising process has been processed. The input is processed from the above step which is the preprocessed image. Gaussian noise and salt peeper noise has been added. The range of noise is between $1-20 \%$. This variability provides us the capability of different level of validation. Then object based data clustering has been performed based on the hierarchical clustering. Decomposition has been performed for the alike element grouping. Based on the similarity ranking and matching similar elements are extracted and processed for the image formation. Then noise extraction process has been started based on the PDE-FFT. This is also called the error. This is extracted based on the mean filter. The resultant data is then obtained based on the noise separation. The edges approach can be helpful in the finding of the adjacent neighborhood. Color domination is helpful in the matching of images based on the color. It provides the noise removal easy as the adjacent position can be determined easily.

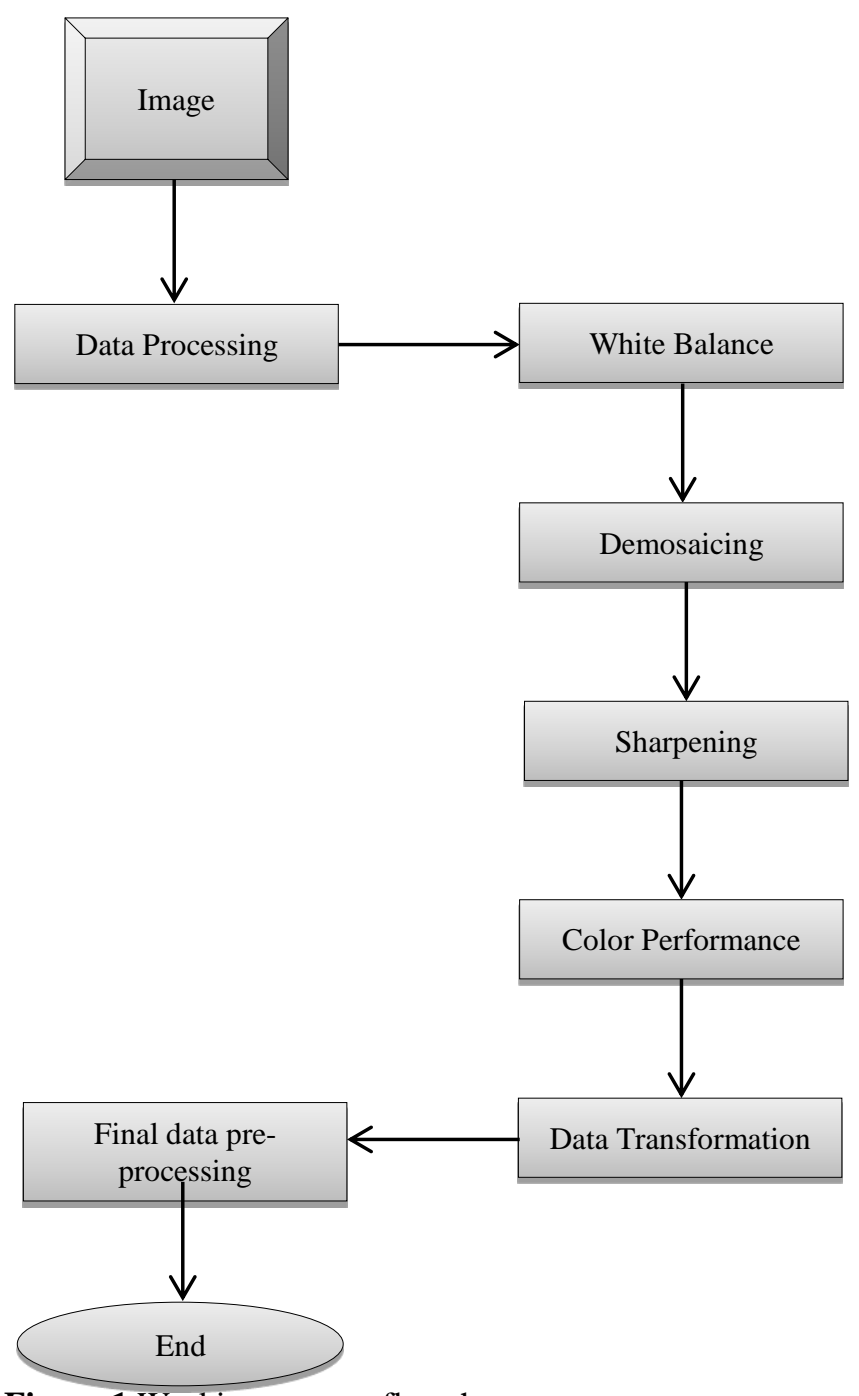

Figure 1 Working process flowchart

\section{Results evaluation}

Different images along with the cameraman images have been considered for the comparative study. PSNR and MSE have been calculated and compared. We have considered Barbara, Leena and Cameraman images. The results clearly depict the improved PSNR results in case of our proposed approach in case of clustered PDE (CPDE) and CPDE + FFT domination (FFTD). 


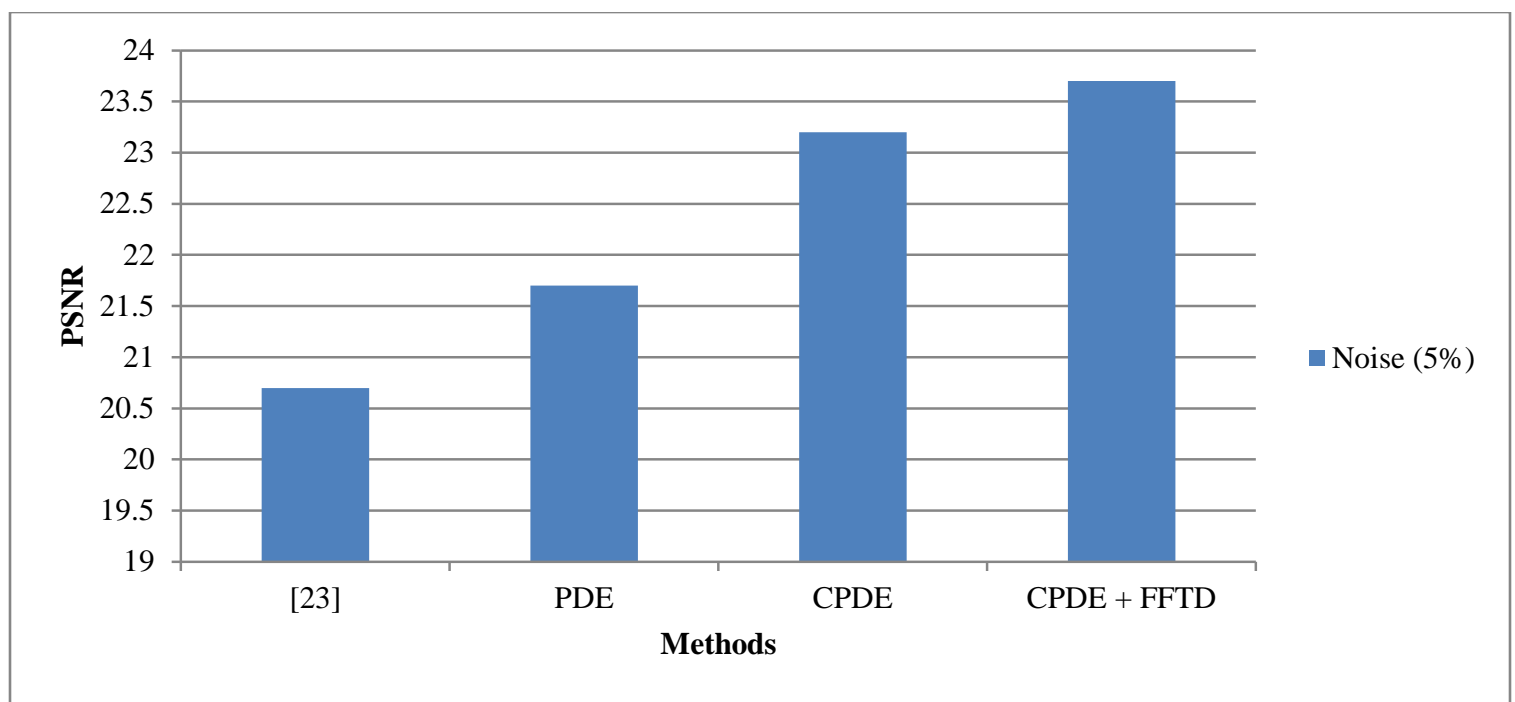

Figure 2 Comparison from the previous and propose approach (Leena)

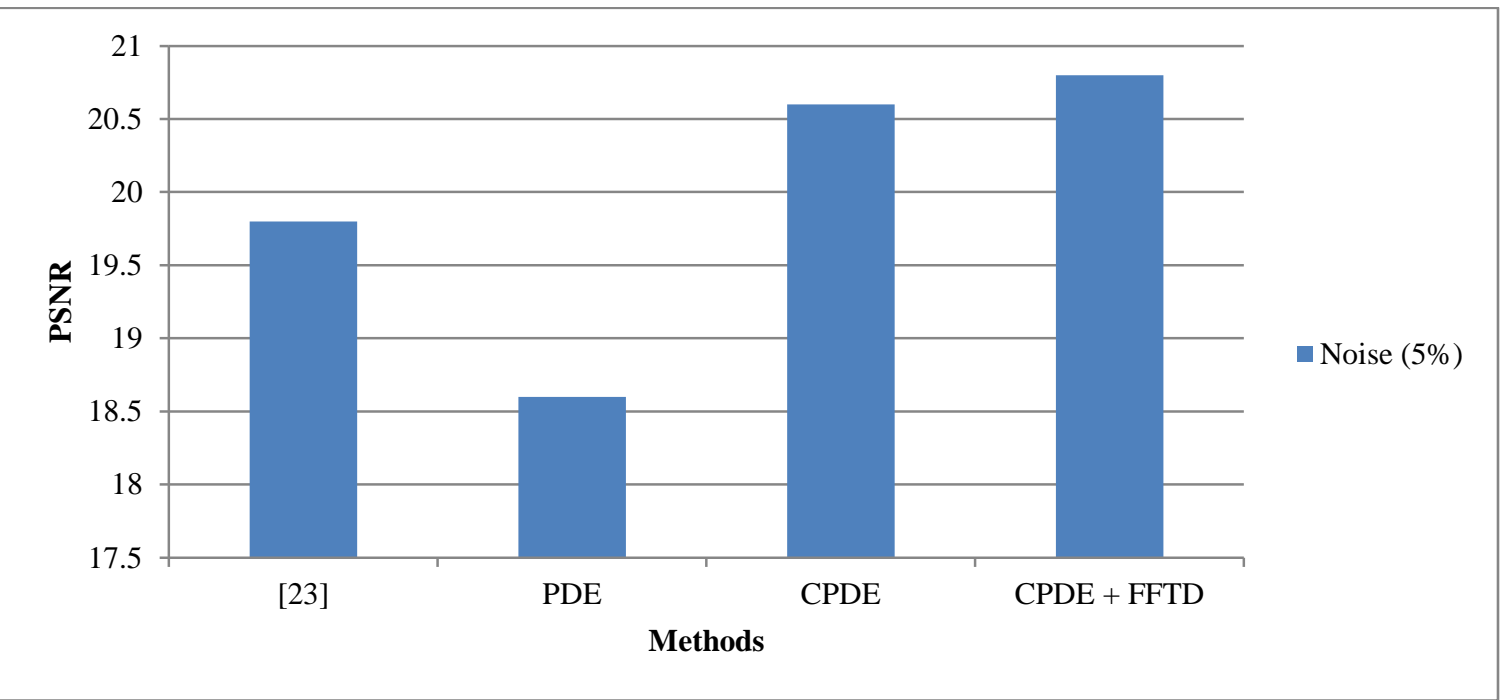

Figure 3 Comparison from the previous and propose approach (Barbara)

\section{Conclusion}

In this dissertation an image denoising method based on clustering and the combination of PDE, FFT and dominates color has been proposed. The work of every method is different so it is applied differently level wise. It has been included terms of clamor rate to discover the effectiveness in terms of PSNR values. The noise percentage considered here is in the range of $1-20 \%$. Hierarchical clustering for the object based data separation has been applied. Then decomposition is performed with the similarity matching and ranking of the image component. The obtained PSNR values suggest that our results are better in comparison to the pervious approach.

\section{Acknowledgment}

None.

\section{Conflicts of interest}

The authors have no conflicts of interest to declare.

\section{References}

[1] Shannon CE. Communication in the presence of noise. Proceedings of the IRE. 1949; 37(1):10-21.

[2] Nyquist H. Certain topics in telegraph transmission theory. Transactions of the American Institute of Electrical Engineers. 1928; 47(2):617-44.

[3] Candès EJ, Wakin MB. An introduction to compressive sampling. IEEE Signal Processing Magazine. 2008; 25(2):21-30. 
[4] Ghosh P, Pandey A, Pati UC. Comparison of different feature detection techniques for image mosaicing. ACCENTS Transactions on Image Processing and Computer Vision. 2015; 1(1):1-7.

[5] Tropp JA, Gilbert AC. Signal recovery from random measurements via orthogonal matching pursuit. IEEE Transactions on Information Theory. 2007; 53(12):4655-66.

[6] Victoria BL, Sathappan S. A survey on impulse noise removal techniques in image processing. International Journal of Advanced Technology and Engineering Exploration. 2018; 5(43):160-4.

[7] Chitra AD, Ponmuthuramalingam P. Face recognition with positive and negative samples using support vector machine. ACCENTS Transactions on Image Processing and Computer Vision. 2016; 2(5): 16-9.

[8] Mohapatra BN, Panda PP. Histogram equalization and noise removal process for enhancement of image. ACCENTS Transactions on Image Processing and Computer Vision. 2017; 3(9): 22-5.

[9] To AC, Moore JR, Glaser SD. Wavelet denoising techniques with applications to experimental geophysical data. Signal Processing. 2009; 89(2):14460.

[10] TV NP, Hemanth VK, Kumar S, Soman KP, Soman A. Comparative study of recent compressed sensing methodologies in astronomical images. In eco-friendly computing and communication systems 2012 (pp. 108-16). Springer, Berlin, Heidelberg.

[11] Dubey S, Hasan F, Shrivastava G. A hybrid method for image denoising based on wavelet thresholding and RBF network. International Journal of Advanced Computer Research. 2012; 2(4): 167-72.

[12] Mohideen SK, Perumal SA, Krishnan N, Selvakumar RK. A novel approach for image denoising using dynamic tracking with new threshold technique. In international conference on computational intelligence and computing research 2010 (pp. 1-4). IEEE.

[13] Benabdelkader S, Soltani O. Wavelet image denoising based spatial noise estimation. In signal processing and intelligent systems conference 2015 (pp. 83-7). IEEE.

[14] Tian J, Chen L. Adaptive image denoising using a non-parametric statistical model of wavelet coefficients. In international symposium on intelligent signal processing and communication systems 2010 (pp. 1-4). IEEE.

[15] Singh J, Dubey RB. Reduction of noise image using LMMSE. International Journal of Advanced Computer Research. 2012; 2(5):147-52.

[16] Anandan P, Sabeenian RS. Curvelet based image compression using support vector machine and core vector machine-a review. International Journal of Advanced Computer Research. 2014; 4(15):675-81.
[17] Veena PV, Devi GR, Sowmya V, Soman KP. Least square based image denoising using wavelet filters. Indian Journal of Science and Technology. 2016; 9(30):1-6.

[18] Rajoriya R, Patidar K, and Chouhan S. A survey and analysis on color image encryption algorithms. ACCENTS Transactions on Information Security. 2018; 3(9):1-5.

[19] Chithra K, Santhanam T. Hybrid denoising technique for suppressing Gaussian noise in medical images. In international conference on power, control, signals and instrumentation engineering 2017 (pp. 1460-3). IEEE.

[20] Soni N, Kirar K. Transform based image denoising: a review. In international conference on recent innovations in signal processing and embedded systems 2017 (pp. 168-71). IEEE.

[21] Pang J. Improved image denoising based on Haar wavelet transform. In smart world, ubiquitous intelligence \& computing, advanced \& trusted computed, scalable computing \& communications, cloud \& big data computing, internet of people and smart city innovation 2017 (pp. 1-6). IEEE.

[22] Yang W, Liu J. Denoising fluorescence molecular image by k-means clustering. In international conference on computer and communications 2017 (pp. 1847-50). IEEE.

[23] Ankarao V, Sowmya V, Soman KP. Sparse image denoising using dictionary constructed based on least square solution. In international conference on wireless communications, signal processing and networking 2017 (pp. 1165-71). IEEE.

[24] Vyas A, Paik J. Applications of multiscale transforms to image denoising: survey. In international conference on electronics, information, and communication 2018 (pp. 1-3). IEEE.

[25] Liu Z, Yan WQ, Yang ML. Image denoising based on a CNN model. In international conference on control, automation and robotics 2018 (pp. 389-93). IEEE.

[26] Mbarki Z, Seddik H, Braiek EB. Non blind image restoration scheme combining parametric wiener filtering and $\mathrm{BM} 3 \mathrm{D}$ denoising technique. In international conference on advanced technologies for signal and image processing 2018 (pp. 1-5). IEEE.

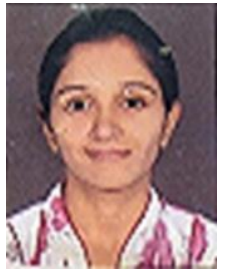

Sonal S. Pandya had completed her $\mathrm{BE}$ in the department of Computer Science and Engineering from Bhagwan Mahavir Collage of Engineering and Technology, Surat, Gujarat in 2013. Currently she is pursuing M.Tech in Computer Science from SRK University, Bhopal, MP.

Email: pandyasonal68@gmail.com 